\title{
Body Composition Measurement Improved Performance of GLIM Criteria in Diagnosing Malnutrition Compared to PG-SGA in Ambulatory Cancer Patients: A Prospective Cross-Sectional Study
}

\author{
Yanfei Wang ${ }^{1}{ }^{(D)}$, Xiaoyan Chen ${ }^{1}$, Yunyi Wang ${ }^{2}$, Ziqi Liu ${ }^{3}$, Yu Fang ${ }^{3}$, Zhi Peng ${ }^{3,4}$ and Wei Liu ${ }^{1,2, *}$ \\ 1 Key Laboratory of Carcinogenesis and Translational Research (Ministry of Education/Beijing), Day Oncology \\ Unit, Peking University Cancer Hospital \& Institute, Beijing 100142, China; yanfeiwang@bjmu.edu.cn (Y.W.); \\ xiaoyan9312@126.com (X.C.) \\ 2 Key Laboratory of Carcinogenesis and Translational Research (Ministry of Education/Beijing), \\ Supportive Care Department, Peking University Cancer Hospital \& Institute, Beijing 100142, China; \\ Beijingwangyunyi@163.com \\ 3 Key Laboratory of Carcinogenesis and Translational Research (Ministry of Education/Beijing), \\ Department of Nutrition, Peking University Cancer Hospital \& Institute, Beijing 100142, China; \\ liuziqi@bjmu.edu.cn (Z.L.); fangyu777@bjmu.edu.cn (Y.F.); zhipeng@bjmu.edu.cn (Z.P.) \\ 4 Key Laboratory of Carcinogenesis and Translational Research (Ministry of Education/Beijing), \\ Department of Gastrointestinal Oncology, Peking University Cancer Hospital \& Institute, \\ Beijing 100142, China \\ check for \\ updates \\ * Correspondence: newbeijingliuwei@163.com; Tel.: +86-010-88196797
}

Citation: Wang, Y.; Chen, X.; Wang, Y.; Liu, Z.; Fang, Y.; Peng, Z.; Liu, W. Body Composition Measurement Improved Performance of GLIM Criteria in Diagnosing Malnutrition Compared to PG-SGA in Ambulatory Cancer Patients: A Prospective Cross-Sectional Study. Nutrients 2021, 13, 2744. https://doi.org/10.3390/ nu13082744

Academic Editors: Roberto Iacone and Ina Bergheim

Received: 21 June 2021

Accepted: 7 August 2021

Published: 10 August 2021

Publisher's Note: MDPI stays neutral with regard to jurisdictional claims in published maps and institutional affiliations.

Copyright: () 2021 by the authors. Licensee MDPI, Basel, Switzerland. This article is an open access article distributed under the terms and conditions of the Creative Commons Attribution (CC BY) license (https:// creativecommons.org/licenses/by/ $4.0 /)$.

\begin{abstract}
Background and aims: Muscle mass reduction (MMR) is one of the three etiologic criteria in the Global Leadership Initiative on Malnutrition (GLIM) framework. This study aimed to evaluate the value of MMR in GLIM criteria among ambulatory cancer patients. Methods: A single-center prospective cross-sectional study was conducted. All participants underwent calf circumference (CC) measurement and body composition measurement by bioelectrical impedance analysis (BIA). MMR was identified by CC, fat-free mass index (FFMI), appendicular skeletal muscle index (ASMI), or combinations of the above three indicators. Patients-generated Subjective Global Assessment (PG-SGA) was used as the comparator. Results: A total of 562 cancer patients receiving intravenous treatment were evaluated. Of the participants, $62.8 \%(355 / 562)$ were male. The median age of the patients was 59.0 years (range, 21-82 year). The median BMI was $22.8 \mathrm{~kg} / \mathrm{m}^{2}$ (range, 14.6-34.5 $\mathrm{kg} / \mathrm{m}^{2}$ ). A total of $41.8 \%$ of patients were evaluated as malnutrition (PG-SGA $\geq 4$ ), and $11.9 \%$ were diagnosed with severe malnutrition (PG-SGA $\geq 9$ ). For the GLIM criteria, the prevalence of malnutrition was $26.9 \%$, and severe malnutrition was $12.3 \%$. For all criteria combinations of GLIM together versus PG-SGA, sensitivity was 60.4\% (53.8-66.7), specificity was 97.9\% (95.4-99.1), while the accordance between GLIM and PG-SGA was moderate $(\kappa=0.614)$. The performance of the GLIM worsened when MMR was excluded $(K=0.515)$, with reduced sensitivity $(50.2 \%(43.7-56.8))$ and the same specificity (97.9\% (95.4-99.1)). Including FFMI and ASMI by BIA can further improve the performance of GLIM than using CC alone ( $k=0.614$ vs. $\kappa=0.565)$. Conclusions: It is important to include MMR in the GLIM framework. Using body composition measurement further improves the performance of the GLIM criteria than using anthropometric measurement alone.
\end{abstract}

Keywords: malnutrition; GLIM; PG-SGA; body composition measurement; muscle mass reduction

\section{Introduction}

Cancer patients are at high risk of malnutrition. It is estimated that approximately $30-90 \%$ of cancer patients suffer from malnutrition due to either the physical and metabolic effects of cancer or the adverse effects of anticancer treatments [1,2]. Malnutrition is associated with reduced treatment effectiveness [3,4], increased treatment toxicities [5], 
impaired functional status and quality of life [6], higher healthcare costs [7], and poorer survival [8-10]. Routine nutritional screening and assessment are suggested in different clinical settings considering the adverse impacts of malnutrition on cancer patients.

Operational diagnostic criteria for the Global Leadership Initiative on Malnutrition (GLIM) were developed from 2016 to 2018 by several global clinical nutrition societies to standardize malnutrition diagnosis in different clinical settings worldwide [11,12]. Since the GLIM criteria are consensus-based, it is necessary to confirm their validity and refine the operational criteria $[13,14]$.

The Patient-generated Subjective Global Assessment (PG-SGA) is a nutrition assessment tool adapted for oncology patients from the Subjective Global Assessment (SGA) tool [15]. It is commonly used among oncology patients worldwide and is recommended by the American Dietetic Association (ADA) [16] and the Chinese Society Oncological Nutrition supportive Care (CSONSC). According to recent published validation guidance on GLIM criteria [13,14], PG-SGA can be used as a secondary reference criterion to diagnose malnutrition in cancer patients.

Muscle mass reduction (MMR) is one of the three phenotypic criteria of the GLIM framework. However, MMR is often omitted in recent literature, which aims to validate GLIM criteria [17,18]. MMR is supposed to be accessed by validated body composition measuring techniques such as fact-free mass index (FFMI) by dual-energy absorptiometry (DXA) or corresponding standards using other body composition methods like bioelectrical impedance analysis (BIA), computed tomography (CT), or magnetic resonance imaging (MRI) [11]. Since the above methods are not often available in most clinical settings for nutritional assessment worldwide, physical examination or anthropometric measures of calf or arm muscle circumference are included as alternative measures [11,12]. To our knowledge, no studies had accessed the difference between using validated body composition measuring techniques, physical examination, or anthropometric measures up to when we constructed this manuscript.

The current study aimed to evaluate the value of MMR in the GLIM framework and compare different methods of defining MMR in an ambulatory clinical setting.

\section{Materials and Methods}

\subsection{Study Design}

Our single-center cross-sectional, observational study was conducted at the Day Oncology Unit in Peking University Cancer Hospital, Beijing, China, spanning 3 weeks from 11 November 2020 to 10 December 2020.

\subsection{Participants}

The eligibility criteria included: (1) diagnosed with cancer by pathology, (2) age $\geq 18 \mathrm{y}$, (3) receiving in-chair intravenous treatment, and (4) having normal cognitive function. Patients were excluded from the study if they were: (1) in poor performance status, namely Eastern Cooperative Oncology Group (ECOG) score > 2; (2) unable to stand up due to illness, or (3) unwilling to participate in the study.

\subsection{Data Collection}

Before the data collection, three doctors and one nutritionist were trained by the same instructor. The training session began with the content and standard procedures of the data collection, followed by a practice session. Any confusion and discrepancies were discussed and solved during the session. The trained doctors and nutritionist completed the data collection by applying a structured questionnaire within $4 \mathrm{~h}$ of hospital admission.

The questionnaire included all items from the PG-SGA and GLIM. Decreased food intake was asked for the past week, 2 weeks, and 1 month based on patients' estimation of reduction in general food intake. Body weight in the past (0.5, 1, 2, 3, or 6 months) was recorded based on patients' self-reporting and electronic medical records (if available), and then the percentage of unintentional weight loss was calculated. 
The collected data also included patients' general information, plasma C-reactive protein (CRP) values, anthropometric measurements, and body composition measurement.

Patients' general information, including age, sex, tumor site, tumor stage, comorbidities and treatment, was obtained from medical records.

CRP was tested using fasting blood, and the values within 1 week of admission were obtained from electrical medical records (if available).

The anthropometric measurement included body weight, height, body mass index (BMI), percentage of weight loss, and calf circumference (CC). The patient's body weight and height were measured in light indoor clothing without shoes at the time of admission. BMI was calculated as the weight $(\mathrm{kg}) /$ height $(\mathrm{m})^{2}$. The CC of both sides were measured using a flexible and non-elastic tape, and the smaller value was recorded for use.

All patients underwent human body composition measurement by BIA using InBody770 (InBody Co., Ltd., Cheonan, Korea) upon entering the ward before receiving intravenous treatment. Inbody770 reports FFMI and appendicular skeletal muscle index (ASMI) values.

The nutritional diagnosis was made during data analysis when PG-SGA and CLIM criteria items were extracted from collected data.

\section{4. $P G-S G A$}

Nutritional status was assessed with the PG-SGA. PG-SGA consists of two sections, namely patient- and clinician-completed components. The patient-completed component includes four aspects: weight loss, nutritional impact symptoms, food intake, and functional capacity. The clinician-completed component assesses four aspects: disease situation, age, metabolic stress, and physical examination. Based on the above assessments, patients were classified as well-nourished (PG-SGA: A, score 0-1), suspected of being or moderately malnourished (PG-SGA: B, score 2-8), or severely malnourished (PG-SGA: C, score $\geq 9$ ) [15]. In order to guide clinical practice, category $B$ can be further divided into suspicious or mild malnutrition (score 2-3) and moderate malnutrition (score 4-8); the latter requires nutritional intervention and symptomatic treatment [19]. In this study, patients with PGSGA score $\geq 4$ were diagnosed with malnutrition, and those with PG-SGA score $\geq 9$ (i.e., category $\mathrm{C}$ ) were identified as having severe malnutrition.

\subsection{GLIM Criteria}

\subsubsection{Step 1: Nutrition Screening}

The malnutrition universal screening tool (MUST) was used to assess malnutrition risk as the first step of the GLIM framework followed by the diagnosis of malnutrition.

The MUST considers parameters including BMI, unintentional weight loss, and acute disease comprising nutritional intake for $>5 \mathrm{~d}$. MUST scores are rated as described in the literature, with a score of 0 indicating low risk, 1 indicating medium risk, and 2 indicating high risk [20]. In this study, patients with MUST scores of medium or high risk were considered at risk of malnutrition and further evaluated by GLIM criteria.

\subsubsection{Step 2: Assessment for Diagnosis}

The GLIM criteria consist of three phenotypic (weight loss, low BMI, and MMR) and two etiologic (reduced food intake/assimilation and disease burden/inflammation) criteria.

\section{Phenotypic Criteria}

In this study, phenotypic criteria were defined as follows:

A. Weight loss: weight loss is defined as unintentional weight loss of $>5 \%$ within the past 6 mo or $>10 \%$ beyond 6 mo.

B. Low BMI: a low BMI for Asians is considered when BMI $<18.5 \mathrm{~kg} / \mathrm{m}^{2}$ if age $<70$ y or $\mathrm{BMI}<20 \mathrm{~kg} / \mathrm{m}^{2}$ if age $>70 \mathrm{y}$. 
C. MMR: In order to evaluate the value of MMR in GLIM criteria and determine the best definition of MMR, the phenotypic criterion MMR was defined using the following eight different definitions, respectively, as follows:

1. "excluded MMR": MMR was not used as a phenotypic criterion.

2. "CC": MMR is defined as $C C<34 \mathrm{~cm}$ in men or CC $<33 \mathrm{~cm}$ for women [21];

3. "FFMI": MMR is identified as FFMI $<17 \mathrm{~kg} / \mathrm{m}^{2}$ in men or $<15 \mathrm{~kg} / \mathrm{m}^{2}$ in women, as established by the European Society for Clinical Nutrition and Metabolism (ESPEN) [22];

4. "ASMI": the cut-off points for diagnosing MMR were set as ASMI $<7.0 \mathrm{~kg} / \mathrm{m}^{2}$ in men or $<5.7 \mathrm{~kg} / \mathrm{m}^{2}$ in women according to the Asian Working Group for Sarcopenia (AWGS) [21];

5. "CC+FFMI": meeting "CC" or "FFMI";

6. "CC+ASMI": meeting "CC" or "ASMI";

7. "FFMI+ASMI": meeting "FFMI" or "ASMI";

8. "CC+FFMI+ASMI": meeting at least one criterion of "CC", "FFMI", and "ASMI".

\section{Etiologic Criteria}

There are two different etiologic criteria in the GLIM criteria. In this study, each etiologic criterion was defined as follows:

A. Reduced intake or assimilation: Reduced intake or assimilation is defined as intake $\leq 50 \%$ of energy requirement for $>1$ week, or reduction for $>2$ weeks, or the presence of disorders which affect assimilation, or gastrointestinal symptoms which were shown in the PG-SGA questionnaire

B. Disease burden or inflammation: Inflammation is identified by plasma C-reactive protein (CRP) $>8 \mathrm{mg} / \mathrm{L}$ (if available; 44 patients were tested for CRP) or current diseases/injury with which inflammation is likely to be associated with $[11,12]$ according to medical records. Since all participants of this study were with malignant disease, malignancy was not used as an indicator for inflammation.

Diagnosis of Malnutrition

To diagnosis malnutrition, at least one phenotypic criterion and at least one etiologic criterion should be met. As for MMR, different definitions were used for the diagnosis of malnutrition.

\subsubsection{Step 3: Severity Grading of GLIM Criteria}

After the diagnosis of malnutrition, the severity of malnutrition is identified by phenotypic criteria. For unintentional weight loss, the cut-off values to grade malnutrition severity were a weight loss of $>10 \%$ within the past 6 months or $>20 \%$ beyond 6 months, as presented in the criteria [11,12]. For low BMI, The cut-off values to grade malnutrition severity were $\mathrm{BMI}<17.0 \mathrm{~kg} / \mathrm{m}^{2}$ if age $<70 \mathrm{y}$ or $\mathrm{BMI}<17.8 \mathrm{~kg} / \mathrm{m}^{2}$ if age $>70 \mathrm{y}$, according to a recent study conducted in a Japanese population [23]. Since there is no consensus on the cut-off value in grading the severity of muscle mass reduction by anthropometric measurements referring to CC, FFMI, and ASMI acquired by BIA, muscle mass reduction was not further divided into different severity grades.

\subsection{Ethics Statement}

The study was approved by the independent institutional ethics committee of the Peking University Cancer Hospital. All enrolled participants signed informed consent for the scientific use of their data.

\subsection{Statistical Analysis}

All analyses were performed using the Statistical Package for the Social Sciences (SPSS), version 20.0 (IBM, Armonk, NY, USA). Results were considered statistically significant when the $p$-value $\leq 0.05$. 
Continuous variables following normal distribution were presented as mean values and standard deviations and otherwise presented as medians and quartiles. Categorical variables were presented as counts and portions.

Statistical evaluations of the CLIM criteria compared to PG-SGA were performed. Sensitivity (SE), specificity (SP), positive predictive value (PPV), and negative predictive value (NPV) were calculated to determine the performance of different combinations of GLIM criteria in light of PG-SGA as the gold standard. Data were expressed as a percent and $95 \%$ confidence interval (CI). Validity statistics were calculated using all possible combinations of the GLIM phenotypic and etiologic criteria; each combination of two criteria, since in some clinical situation, not all data for each criterion were accessible. Comparisons were made to the criterion of malnutrition (PG-SGA score $\geq 4$ ) or severe malnutrition (PG-SGA score $\geq 9$ ). The agreement among the GLIM and PG-SGA was addressed by $\kappa . \kappa$ that is $>0.80$ is substantial, whereas $0.61-0.80$ is moderate, the presence of lower $\kappa$ values brings into question the reliability of the GLIM criteria [14,24]. The rating of validity test statistics also followed recommended cut points for sensitivity and specificity: both SE and SP $>80 \%$ is 'good'; SE or SP $>80 \%$ and both $>50 \%$ is 'fair'; SE or SP $>50 \%$ is 'poor' [17,25].

\section{Results}

\subsection{Characteristics of Participants}

A total of 686 patients were admitted to day oncology from 11 November 2020 to 10 December 2020, and 562 cancer patients participated in this study (see flow chart, Figure 1).

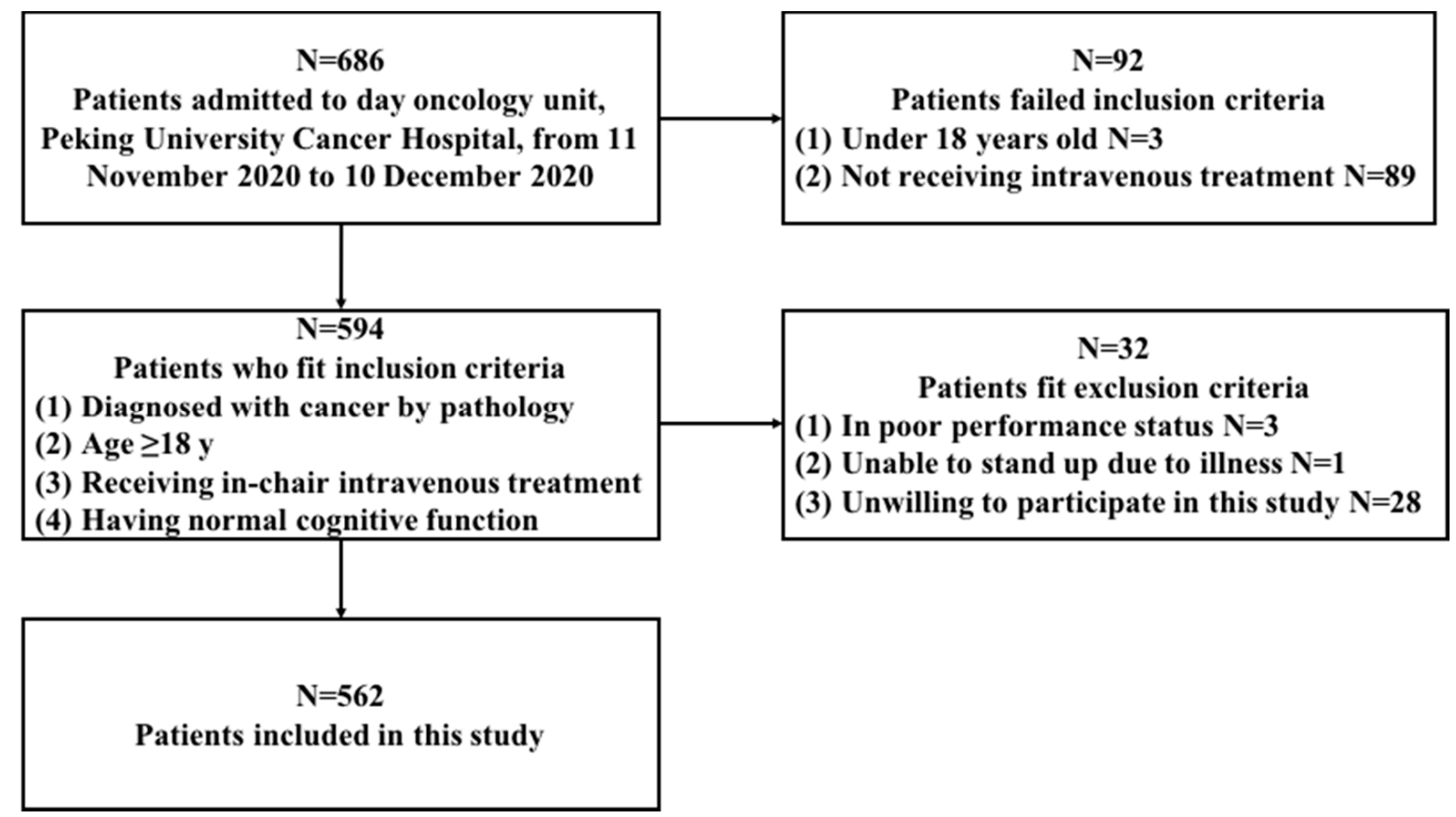

Figure 1. Flow chart.

Of the participants, $62.8 \%(355 / 562)$ were male, and 37.2\% (210/562) were female, with a male to female ratio of 1.69:1. The median age of the patients was 59.0 years (range, 21-82 y; the interquartile range was 52.0-65.0 y). The median BMI was $22.8 \mathrm{~kg} / \mathrm{m}^{2}$ (range, $14.6-34.5 \mathrm{~kg} / \mathrm{m}^{2}$; the interquartile range was $\left.20.5-25.2 \mathrm{~kg} / \mathrm{m}^{2}\right)$. The characteristics of the patients are displayed in Table 1. 
Table 1. Characteristics of 562 patients with cancer enrolled in this study.

\begin{tabular}{cc}
\hline Parameter & Total $\boldsymbol{n}=5 \mathbf{6 2}$ \\
\hline Age, y (median, interquartile range) & $59(52-65)$ \\
Sex, n (\%) & \\
Male & $354(70.3)$ \\
Female & $208(37.0)$ \\
Tumor site, $\mathrm{n}(\%)$ & \\
Upper Gastrointestinal tract & $134(23.8)$ \\
Colorectal & $291(51.8)$ \\
Head and neck & $1(0.2)$ \\
Lung and mediastinum & $43(7.7)$ \\
Breast & $21(3.7)$ \\
Urogenital & $11(2.0)$ \\
Gynecology & $1(0.2)$ \\
Liver, pancreas & $40(7.1)$ \\
Lymphoma & $4(0.7)$ \\
Other & $16(2.8)$ \\
Tumor stage, $\mathrm{n}(\%)$ & $10(1.8)$ \\
I & $45(8.0)$ \\
II & $176(31.3)$ \\
III & $331(58.9)$ \\
IV &
\end{tabular}

\subsection{Nutritional Screening and Evaluation Results of the Participants}

Of the 562 patients, the prevalence of malnutrition as per scored PG-SGA $\geq 4$ was $41.8 \%(235 / 562)$, from which severe malnutrition (scored PG-SGA $\geq 9$, or PG-SGA C) was $11.9 \%(67 / 562)$.

The prevalence of patients at malnutrition risk, namely MUST moderate or high risk, was $59.8 \%(336 / 562)$. Patients screened as a malnutrition risk were further evaluated by the GLIM criteria.

As for the GLIM criteria, the prevalence of participants meeting each criterion is shown in Table 2. The prevalence of malnutrition diagnosed by different combinations of phenotypic and etiologic criteria is shown in Table 3.

Table 2. The prevalence of patients meeting each criterion of the GLIM framework.

\begin{tabular}{|c|c|c|}
\hline \multicolumn{2}{|c|}{ Criteria } & $n(\%)$ \\
\hline \multicolumn{2}{|l|}{ Phenotypic Criteria } & Total $n=562$ \\
\hline \multirow{2}{*}{\multicolumn{2}{|c|}{$\begin{array}{l}\text { Weight loss } \\
\text { Low BMI }\end{array}$}} & $227(40.4)$ \\
\hline & & $60(10.7)$ \\
\hline \multirow{7}{*}{ MMR } & $\mathrm{CC}$ & $129(23.0)$ \\
\hline & FFMI & $158(28.1)$ \\
\hline & ASMI & $119(21.2)$ \\
\hline & CC+FFMI & $194(34.5)$ \\
\hline & CC+ASMI & $162(28.8)$ \\
\hline & FFMI+ASMI & $168(29.9)$ \\
\hline & $\mathrm{CC}+\mathrm{FFMI}+\mathrm{ASMI}$ & $197(35.1)$ \\
\hline \multicolumn{2}{|c|}{ Etiologic criteria } & Total $n=562$ \\
\hline \multicolumn{2}{|c|}{ Reduced intake or assimilation } & $186(33.1)$ \\
\hline \multirow{3}{*}{$\begin{array}{l}\text { Disease burden or } \\
\text { inflammation }\end{array}$} & $\begin{array}{l}\text { Inflammation-associated } \\
\text { disease or injury }\end{array}$ & $0(0)$ \\
\hline & & Total $n=44$ \\
\hline & Elevated plasm CRP & $7(1.2)$ \\
\hline
\end{tabular}


Table 3. The prevalence of malnutrition diagnosed by different combinations of phenotypic and etiologic criteria of the GLIM framework.

\begin{tabular}{|c|c|}
\hline Combination & Prevalence $n(\%)$ \\
\hline $\begin{array}{l}\text { Weight loss } \\
\text { Reduced intake or assimilation }\end{array}$ & $117(20.8)$ \\
\hline $\begin{array}{l}\text { Low BMI } \\
\text { Reduced intake or assimilation }\end{array}$ & $25(4.4)$ \\
\hline $\begin{array}{l}\text { MMR * } \\
\text { Reduced intake or assimilation }\end{array}$ & $87(15.5)$ \\
\hline $\begin{array}{c}\text { Any phenotypic criterion } \\
\text { Reduced intake or assimilation }\end{array}$ & $148(26.3)$ \\
\hline $\begin{array}{c}\text { Weight loss } \\
\text { Disease burden/inflammation }\end{array}$ & $1(2.3)$ \\
\hline $\begin{array}{c}\text { Low BMI } \\
\text { Disease burden/inflammation }\end{array}$ & $0(0)$ \\
\hline $\begin{array}{c}\text { MMR * } \\
\text { Disease burden/inflammation }\end{array}$ & $0(0)$ \\
\hline $\begin{array}{c}\text { Any phenotypic criterion } \\
\text { Disease burden/inflammation }\end{array}$ & $44(2.3)$ \\
\hline $\begin{array}{c}\text { Weight loss } \\
\text { Any etiologic criterion }\end{array}$ & $118(21.0)$ \\
\hline $\begin{array}{c}\text { Low BMI } \\
\text { Any etiologic criterion }\end{array}$ & $25(4.4)$ \\
\hline $\begin{array}{l}\text { MMR * } \\
\text { Any etiologic criterion }\end{array}$ & 87 (15.5) \\
\hline $\begin{array}{l}\text { Any phenotypic criterion } \\
\text { Any etiologic criterion }\end{array}$ & $149(26.5)$ \\
\hline
\end{tabular}

Using all combinations of the above phenotypic and etiologic criteria, the prevalence of malnutrition diagnosed by the GLIM criteria was $26.5 \%(149 / 562)$, with a prevalence of severe malnutrition at $12.3 \%(69 / 562)$. Compare with PG-SGA, GLIM criteria underrepresented malnutrition while overrating severe malnutrition.

\subsection{Comparision between the GLIM Criteria Using Different Definitions of MMR and the PG-SGA}

Using any phenotypic criteria combined with etiologic criteria yielded greater SE and $\kappa$ compared with PG-SGA than using a single phenotypic criterion alone combined with etiologic criteria (shown in Table A1). Therefore, we compared GLIM framework in any combination of phenotypic and etiologic criteria to PG-SGA, so as to evaluate the value of MMR assessment in GLIM criteria.

Table 4 shows the SE, SP, PPV, and NPV of any combination of phenotypic and etiologic criteria of the GLIM framework in diagnosing malnutrition compared with PG-SGA. Incorporating MMR into the phenotypic criteria increased the SE of GLIM criteria while SP remained the same regardless of what definition of reduced mass was used. For different definitions of MMR, using FFMI to identify MMR had better accordance with PG-SGA in diagnosing malnutrition than using CC or ASMI $(\kappa=0.598, \kappa=0.565, k=0.586$, respectively, Table 4). Any combination of the indicators (CC+FFMI, CC+ASMI, FFMI+ASMI, and $\mathrm{CC}+\mathrm{FFMI}+\mathrm{ASMI}$ ) further improved performance compared with using one indicator alone. When we combined CC, FFSMI, and ASMI to define MMR, the GLIM framework had the best performance compared with PG-SGA, which had an SE of $60.4 \%$ (53.8-66.7), SP of $97.9 \%$ (95.4-99.1), and accordance with the PG-SGA was moderate $(\kappa=0.614)$. 
Table 4. SE, SP, PPV, and NPV of GLIM using any combination of phenotypic criteria and etiologic criteria in diagnosing malnutrition compared with PG-SGA.

\begin{tabular}{|c|c|c|c|c|c|}
\hline Definition of MMR & $\begin{array}{c}\mathrm{SE} \\
(\%, 95 \% \mathrm{CI})\end{array}$ & $\begin{array}{c}\text { SP } \\
(\%, 95 \% \mathrm{CI})\end{array}$ & $\begin{array}{c}\text { PPV } \\
(\%, 95 \% \mathrm{CI})\end{array}$ & $\begin{array}{c}\text { NPV } \\
(\%, 95 \% \mathrm{CI})\end{array}$ & K \\
\hline Excluded MMR & $50.2(43.7-56.8)$ & $97.9(95.4-99.1)$ & $94.4(88.4-97.5)$ & $73.2(68.8-77.3)$ & 0.515 \\
\hline $\mathrm{CC}$ & $55.3(48.7-61.7)$ & 97.9 (95.4-99.1) & $94.9(89.4-97.8)$ & $75.3(70.9-79.3)$ & 0.565 \\
\hline FFMI & $58.7(52.1-65.0)$ & $97.9(95.4-99.1)$ & $95.2(90.0-97.9)$ & $76.7(72.3-80.7)$ & 0.598 \\
\hline ASMI & $57.4(50.8-63.8)$ & 97.9 (95.4-99.1) & 95.1 (89.7-97.8) & $76.2(71.8-80.1)$ & 0.586 \\
\hline $\mathrm{CC}+\mathrm{FFMI}$ & $60.0(53.4-66.3)$ & $97.9(95.4-99.1)$ & 95. 3 (90.1-97.9) & $77.3(72.9-81.2)$ & 0.610 \\
\hline CC+ASMI & $59.1(52.6-65.4)$ & $97.9(95.4-99.1)$ & $95.2(90.0-97.9)$ & $76.9(72.5-80.8)$ & 0.602 \\
\hline FFMI+ASMI & $59.1(52.6-65.4)$ & $97.9(95.4-99.1)$ & $95.2(90.0-97.9)$ & $76.9(72.5-80.8)$ & 0.602 \\
\hline $\mathrm{CC}+\mathrm{FFMI}+\mathrm{ASMI}$ & $60.4(53.8-66.7)$ & 97.9 (95.4-99.1) & $95.3(90.2-98.0)$ & $77.5(73.1-81.4)$ & 0.614 \\
\hline
\end{tabular}

MMR: muscle mass reduction; SE: sensitivity; SP: specificity; PPV: positive predictive value; NPV: negative predictive value; CI: confidence interval; PG-SGA: Patient-generated Subjective Global Assessment; CC: calf circumference; FFMI: fat-free mass index; ASMI: appendicular skeletal muscle index.

For diagnosing of severe malnutrition, excluding MMR or using different definitions to define MMR in GLIM criteria yielded the same results referring to SE (58.2\% (45.5-69.9)), SP (93.9\% (91.4-95.8)), PPV (56.5\% (44.1-68.2)) and NPV (94.3\% (91.8-96.1)), as well as the accordance to PG-SGA $(\kappa=0.515)$. (Table 5).

Table 5. SE, SP, PPV and NPV of GLIM using any combination of phenotypic and etiologic criteria in diagnosing severe malnutrition compared with PG-SGA.

\begin{tabular}{|c|c|c|c|c|c|}
\hline Definition of MMR & $\begin{array}{c}\text { SE } \\
(\%, 95 \% C I)\end{array}$ & $\begin{array}{c}\text { SP } \\
(\%, 95 \% \mathrm{CI})\end{array}$ & $\begin{array}{c}\text { PPV } \\
(\%, 95 \% \mathrm{CI})\end{array}$ & $\begin{array}{c}\text { NPV } \\
(\%, 95 \% \mathrm{CI})\end{array}$ & к \\
\hline Excluded MMR & $58.2(45.5-69.9)$ & 93.9 (91.4-95.8) & $56.5(44.1-68.2)$ & $94.3(91.8-96.1)$ & 0.515 \\
\hline $\mathrm{CC}$ & $58.2(45.5-69.9)$ & 93.9 (91.4-95.8) & $56.5(44.1-68.2)$ & $94.3(91.8-96.1)$ & 0.515 \\
\hline FFMI & $58.2(45.5-69.9)$ & 93.9 (91.4-95.8) & $56.5(44.1-68.2)$ & $94.3(91.8-96.1)$ & 0.515 \\
\hline ASMI & $58.2(45.5-69.9)$ & 93.9 (91.4-95.8) & $56.5(44.1-68.2)$ & $94.3(91.8-96.1)$ & 0.515 \\
\hline CC+FFMI & $58.2(45.5-69.9)$ & 93.9 (91.4-95.8) & $56.5(44.1-68.2)$ & $94.3(91.8-96.1)$ & 0.515 \\
\hline $\mathrm{CC}+\mathrm{ASMI}$ & $58.2(45.5-69.9)$ & $93.9(91.4-95.8)$ & $56.5(44.1-68.2)$ & $94.3(91.8-96.1)$ & 0.515 \\
\hline FFMI+ASMI & $58.2(45.5-69.9)$ & 93.9 (91.4-95.8) & $56.5(44.1-68.2)$ & $94.3(91.8-96.1)$ & 0.515 \\
\hline $\mathrm{CC}+\mathrm{FFMI}+\mathrm{ASMI}$ & $58.2(45.5-69.9)$ & $93.9(91.4-95.8)$ & $56.5(44.1-68.2)$ & $94.3(91.8-96.1)$ & 0.515 \\
\hline
\end{tabular}

MMR: muscle mass reduction; SE: sensitivity; SP: specificity; PPV: positive predictive value; NPV: negative predictive value; CI: confidence interval; PG-SGA: Patient-generated Subjective Global Assessment; CC: calf circumference; FFMI: fat-free mass index; ASMI: appendicular skeletal muscle index.

\section{Discussion}

Since the publication of the GLIM criteria in 2019, there have been emerging studies assessing the validity of the GLIM framework for diagnosing malnutrition. A big problem for validation is that there are currently no worldwide gold standards for diagnosing malnutrition. An in-depth nutrition assessment completed by experienced nutrition experts could be regarded as the semi-gold standard for validating the GLIM framework [13,14], but the specific procedures requested were still uncertain. For now, standardized tools which have been validated, such as SGA, PG-SGA, or Mini Nutritional Assessment (MNA), are still the most commonly used standard for determining the validity of GLIM $[13,14,17]$. Since PG-SGA is specially designed for cancer patients and has been used worldwide, we chose PG-SGA as the comparator to evaluate the performance of GLIM using different definitions of criteria.

Previous studies revealed that two-item single combinations of GLIM criteria are insufficient to diagnose malnutrition, while combining any two etiologic or phenotypic criteria greatly improves sensitivity [17], which is consistent with our result shown in Table A1. There has been strong evidence to support MMR, as one of the three phenotypic criteria, to be included in GLIM criteria. However, there is no consensus regarding measuring and defining MMR, particularly in clinical settings [11,12]. 
In published studies validating GLIM, MMR was not often included in phenotypic criteria due to its retrospective nature or condition restrictions $[17,18]$, while in some other studies, mid-arm muscle circumstance or hand-grip strength were used to represent muscle mass reduction $[9,26,27]$. No studies have provided reliable body composition measurement data to validate the GLIM criteria, and it had been assumed that including FFMI into the GLIM criteria may change the SE and SP [17]. Moreover, recent literature has revealed that cancer patients with reduced FFMI identified by BIA had shorter survival (14.0 months vs. 45.1 months; $p<0.001)$ and worse quality of life than the cancer patients with normal FFMI [28], which proved the importance of assessing MMR by BIA. In this study, we applied BIA in every participant and provided precise information on MMR.

Our study reveals that any indicator of muscle mass evaluation (CC, FFMI, ASMI) increased sensitivity of diagnosing malnutrition compared with the GLIM criteria that excluded MMR, yielding better accordance with PG-SGA; this indicated the importance of muscle mass evaluation, even using simple methods. While body composition measurement facilities such as DXA or BIA are not available in most medical centers, reliable objective and feasible measures can be used as a substitute.

Despite the improvement in diagnosing malnutrition, including MMR assessment failed to improve the SE, SP, PPV, or NPV of GLIM criteria in diagnosing severe malnutrition; this might be explained by the fact that we did not further divide the MMR into different severity grading due to the lack of well-accepted cut-off points. Studies aiming to determine the cut-off points to grade MMR severities are warranted.

Body composition measurement applied by BIA is more reliable than anthropometry measurements not only because it is a comprehensive evaluation of the body but also because it is an objective measure and evades interobserver error. Unsurprisingly, our study showed that either FFMI or ASMI yielded better results than CC, while the FFMI+ASMI combination further improved the sensitivity of GLIM. However, the best performance of GLIM criteria is acquired by combining body composition measurement (FFMI+ASMI) and anthropometry measurement $(\mathrm{CC})$ together $(\mathrm{K}=0.614)$. Future study needs to be done in a wider population to determine the best method to define MMR.

Our study has some strengths. As a prospective cross-sectional observational study, we recruited 562 patients in a short period of time (3 weeks) and all data for PG-SGA and GLIM were collected concurrently, which ensured that the conclusion drawn from the study was relatively more reliable. Moreover, we applied BIA in every participant and provided a precise evaluation of MMR. To the best of our knowledge, this is the first study including muscle mass evaluation by BIA to validate the GLIM criteria.

Nonetheless, there are several limitations to our study. Since it was a single-center observational study, results should be interpreted with caution. Food intake was assessed via self-report on the amount of food instead of an in-depth diet history to promote feasibility. Inflammation was assessed by increased CRP and current inflammation-associated disease or injury. However, the detection rate of CRP was low in our study since no patients presented diseases or injuries likely to be associated with inflammation except for malignant disease, and CRP was not a regular detected indicator for ambulatory cancer patients in our center; therefore, the evaluation of inflammation status is inadequate.Finally, we must contact the participant again to collect survival data revealing the value of the GLIM criteria in predicting clinical outcomes and survival.

\section{Conclusions}

In conclusion, the prevalence of malnutrition is high among ambulatory cancer patients. Using PG-SGA as the standard, GLIM criteria had fair criterion validity for the diagnosis of malnutrition, regardless of severity status. It is important to include MMR in the GLIM framework, while body composition measurement applied by BIA further improves the performance of GLIM criteria. When validated facilities were not available, simple anthropometric measurements can be used. 
Author Contributions: Conceptualization, Y.W. (Yanfei Wang), Y.F., Z.P., and W.L.; methodology, Y.W. (Yanfei Wang); software, Y.W. (Yanfei Wang); validation, X.C., Y.W. (Yunyi Wang), and Z.L.; formal analysis, Y.W. (Yanfei Wang), X.C., Y.W. (Yunyi Wang), and Z.L.; investigation, Y.W. (Yanfei Wang); X.C.; Y.W. (Yunyi Wang), and Z.L.; data curation, Y.W. (Yanfei Wang), X.C., and Y.W. (Yunyi Wang); writing-original draft preparation, Y.W. (Yanfei Wang); writing-review and editing, X.C.; Y.W. (Yunyi Wang); Z.L., Y.F., Z.P., and W.L.; project administration, Y.F., Z.P., and W.L.; funding acquisition, W.L. and Y.W. (Yanfei Wang). All authors have read and agreed to the published version of the manuscript.

Funding: This research was funded by the Beijing Municipal Administration of Hospitals' Mission Plan (to Wei LIU), grant number SML20181102, the Leading Talents of Science and Technology Innovation in the National "Ten thousand Talents Program" (to Wei LIU), and the Science Foundation of Peking University Cancer Hospital (to Yanfei Wang), grant number 2021-23.

Institutional Review Board Statement: The study was conducted according to the guidelines of the Declaration of Helsinki and approved by the independent institutional ethics committee of the Peking University Cancer Hospital (protocol code: 2020KT103, date of approval: 30 October 2020).

Informed Consent Statement: Informed consent was obtained from all subjects involved in the study.

Acknowledgments: We sincerely thank Xu Wang and Ziran Yu for performing the anthropometric measurements and body composition measurement in this study.

Conflicts of Interest: The authors declare no conflict of interest.

\section{Appendix A}

Table A1. SE, SP, PPV, and NPV of GLIM using different combinations of phenotypic criteria and etiologic criteria in diagnosing malnutrition compared with PG-SGA.

\begin{tabular}{|c|c|c|c|c|c|}
\hline Combination & $\begin{array}{c}\mathrm{SE} \\
(\%, 95 \% \mathrm{CI})\end{array}$ & $\begin{array}{c}\mathrm{SP} \\
(\%, 95 \% \mathrm{CI})\end{array}$ & $\begin{array}{c}\text { PPV } \\
(\%, 95 \% \mathrm{CI})\end{array}$ & $\begin{array}{c}\text { NPV } \\
(\%, 95 \% \mathrm{CI})\end{array}$ & к \\
\hline $\begin{array}{c}\text { Weight loss } \\
\text { Reduced intake or assimilation }\end{array}$ & $48.5(42.1-55.0)$ & 98.2 (95.9-99.2) & $95.1(89.2-98.0)$ & $72.1(67.7-76.2)$ & 0.488 \\
\hline $\begin{array}{l}\text { Low BMI } \\
\text { Reduced intake or assimilation }\end{array}$ & $10.2(6.8,15.0)$ & 99.7 (98.0-100.0) & $96.0(77.7-99.8)$ & $60.7(56.4-64.8)$ & 0.113 \\
\hline $\begin{array}{c}\text { MMR * } \\
\text { Reduced intake or assimilation }\end{array}$ & $36.2(30.1-42.7)$ & 99.4 (97.6-99.9) & 97.7 (91.2-99.6) & $68.4(64.0-72.5)$ & 0.390 \\
\hline $\begin{array}{c}\text { Any phenotypic criterion } \\
\text { Reduced intake or assimilation }\end{array}$ & $60.4(53.8-66.7)$ & $98.2(95.8-99.2)$ & $95.9(91.0-98.3)$ & $77.5(73.1-81.4)$ & 0.618 \\
\hline $\begin{array}{c}\text { Weight loss } \\
\text { Disease burden/inflammation }\end{array}$ & $0(0-2.0)$ & $99.7(98.0-100.0)$ & $0(0-94.5)$ & $58.1(53.9-62.2)$ & -0.004 \\
\hline $\begin{array}{c}\text { Low BMI } \\
\text { Disease burden/inflammation }\end{array}$ & $0(0-2.0)$ & $100.0(98.6-100.0)$ & - & - & 0.000 \\
\hline $\begin{array}{c}\text { MMR * } \\
\text { Disease burden/inflammation }\end{array}$ & $0(0-2.0)$ & $100.0(98.6-100.0)$ & - & - & 0.000 \\
\hline $\begin{array}{l}\text { Any phenotypic criterion } \\
\text { Disease burden/inflammation }\end{array}$ & $0(0-2.0)$ & 99.7 (98.0-100.0) & $0(0-94.5)$ & $58.1(53.9-62.2)$ & -0.004 \\
\hline $\begin{array}{l}\text { Weight loss } \\
\text { Any etiologic criterion }\end{array}$ & $47.2(40.7-53.8)$ & $97.9(95.4-99.1)$ & $94.1(87.8-97.4)$ & $72.1(67.6-76.1)$ & 0.485 \\
\hline $\begin{array}{l}\text { Low BMI } \\
\text { Any etiologic criterion }\end{array}$ & $10.2(6.8-15.0)$ & 99.7 (98.0-100.0) & $96.0(77.7-99.8)$ & $60.7(56.4-64.8)$ & 0.113 \\
\hline $\begin{array}{c}\text { MMR * } \\
\text { Any etiologic criterion }\end{array}$ & $36.2(30.1-42.7)$ & 99.4 (97.6-99.9) & 97.7 (91.2-99.6) & $68.4(64.0-72.5)$ & 0.39 \\
\hline $\begin{array}{l}\text { Any phenotypic criterion } \\
\text { Any etiologic criterion }\end{array}$ & $60.4(53.8-66.7)$ & $97.9(95.4-99.1)$ & $95.3(90.2-97.9)$ & $77.5(73.1-81.4)$ & 0.614 \\
\hline
\end{tabular}

SE: sensitivity; SP: specificity; PPV: positive predictive value; NPV: negative predictive value; CI: confidence interval; PG-SGA: Patientgenerated Subjective Global Assessment; BMI: body mass index; MMR: muscle mass reduction. * MMR in this table was defined by the combination of CC, FFMI, or ASMI. 


\section{References}

1. Hebuterne, X.; Lemarie, E.; Michallet, M.; de Montreuil, C.B.; Schneider, S.M.; Goldwasser, F. Prevalence of malnutrition and current use of nutrition support in patients with cancer. JPEN J. Parenter Enter. Nutr. 2014, 38, 196-204. [CrossRef]

2. Arends, J.; Baracos, V.; Bertz, H.; Bozzetti, F.; Calder, P.C.; Deutz, N.E.P.; Erickson, N.; Laviano, A.; Lisanti, M.P.; Lobo, D.N.; et al. ESPEN expert group recommendations for action against cancer-related malnutrition. Clin. Nutr. 2017, 36, 1187-1196. [CrossRef]

3. Salas, S.; Deville, J.L.; Giorgi, R.; Pignon, T.; Bagarry, D.; Barrau, K.; Zanaret, M.; Giovanni, A.; Bourgeois, A.; Favre, R.; et al. Nutritional factors as predictors of response to radio-chemotherapy and survival in unresectable squamous head and neck carcinoma. Radiother. Oncol. 2008, 87, 195-200. [CrossRef] [PubMed]

4. Arends, J.; Bachmann, P.; Baracos, V.; Barthelemy, N.; Bertz, H.; Bozzetti, F.; Fearon, K.; Hutterer, E.; Isenring, E.; Kaasa, S.; et al. ESPEN guidelines on nutrition in cancer patients. Clin. Nutr. 2017, 36, 11-48. [CrossRef] [PubMed]

5. Hill, A.; Kiss, N.; Hodgson, B.; Crowe, T.C.; Walsh, A.D. Associations between nutritional status, weight loss, radiotherapy treatment toxicity and treatment outcomes in gastrointestinal cancer patients. Clin. Nutr. 2011, 30, 92-98. [CrossRef] [PubMed]

6. Tian, J.; Chen, J.S. Nutritional status and quality of life of the gastric cancer patients in Changle County of China. World J. Gastroenterol. 2005, 11, 1582-1586. [CrossRef]

7. Planas, M.; Alvarez-Hernandez, J.; Leon-Sanz, M.; Celaya-Perez, S.; Araujo, K.; Garcia de Lorenzo, A.; Researchers, P.R. Prevalence of hospital malnutrition in cancer patients: A sub-analysis of the PREDyCES(R) study. Support. Care Cancer 2016, $24,429-435$. [CrossRef]

8. Rondel, A.; Langius, J.A.E.; de van der Schueren, M.A.E.; Kruizenga, H.M. The new ESPEN diagnostic criteria for malnutrition predict overall survival in hospitalised patients. Clin. Nutr. 2018, 37, 163-168. [CrossRef] [PubMed]

9. Contreras-Bolivar, V.; Sanchez-Torralvo, F.J.; Ruiz-Vico, M.; Gonzalez-Almendros, I.; Barrios, M.; Padin, S.; Alba, E.; Olveira, G. GLIM Criteria Using Hand Grip Strength Adequately Predict Six-Month Mortality in Cancer Inpatients. Nutrients 2019, 11, 2043. [CrossRef]

10. Lin, J.; Peng, J.; Qdaisat, A.; Li, L.; Chen, G.; Lu, Z.; Wu, X.; Gao, Y.; Zeng, Z.; Ding, P.; et al. Severe weight loss during preoperative chemoradiotherapy compromises survival outcome for patients with locally advanced rectal cancer. J. Cancer Res. Clin. Oncol. 2016, 142, 2551-2560. [CrossRef]

11. Jensen, G.L.; Cederholm, T.; Correia, M.; Gonzalez, M.C.; Fukushima, R.; Higashiguchi, T.; de Baptista, G.A.; Barazzoni, R.; Blaauw, R.; Coats, A.J.S.; et al. GLIM Criteria for the Diagnosis of Malnutrition: A Consensus Report From the Global Clinical Nutrition Community. JPEN J. Parenter Enter. Nutr. 2019, 43, 32-40. [CrossRef] [PubMed]

12. Cederholm, T.; Jensen, G.L.; Correia, M.; Gonzalez, M.C.; Fukushima, R.; Higashiguchi, T.; Baptista, G.; Barazzoni, R.; Blaauw, R.; Coats, A.; et al. GLIM criteria for the diagnosis of malnutrition-A consensus report from the global clinical nutrition community. Clin. Nutr. 2019, 38, 1-9. [CrossRef] [PubMed]

13. de van der Schueren, M.A.E.; Keller, H.; Consortium, G.; Cederholm, T.; Barazzoni, R.; Compher, C.; Correia, M.; Gonzalez, M.C.; Jager-Wittenaar, H.; Pirlich, M.; et al. Global Leadership Initiative on Malnutrition (GLIM): Guidance on validation of the operational criteria for the diagnosis of protein-energy malnutrition in adults. Clin. Nutr. 2020, 39, 2872-2880. [CrossRef] [PubMed]

14. Keller, H.; de van der Schueren, M.A.E.; Consortium, G.; Jensen, G.L.; Barazzoni, R.; Compher, C.; Correia, M.; Gonzalez, M.C.; Jager-Wittenaar, H.; Pirlich, M.; et al. Global Leadership Initiative on Malnutrition (GLIM): Guidance on Validation of the Operational Criteria for the Diagnosis of Protein-Energy Malnutrition in Adults. JPEN J. Parenter Enter. Nutr. 2020, 44, $992-1003$. [CrossRef] [PubMed]

15. Ottery, F.D. Definition of standardized nutritional assessment and interventional pathways in oncology. Nutrition 1996, 12, S15-S19. [CrossRef]

16. Thompson, K.L.; Elliott, L.; Fuchs-Tarlovsky, V.; Levin, R.M.; Voss, A.C.; Piemonte, T. Oncology Evidence-Based Nutrition Practice Guideline for Adults. J. Acad. Nutr. Diet. 2017, 117, 297-310.e247. [CrossRef] [PubMed]

17. Allard, J.P.; Keller, H.; Gramlich, L.; Jeejeebhoy, K.N.; Laporte, M.; Duerksen, D.R. GLIM criteria has fair sensitivity and specificity for diagnosing malnutrition when using SGA as comparator. Clin. Nutr. 2020, 39, 2771-2777. [CrossRef]

18. De Groot, L.M.; Lee, G.; Ackerie, A.; van der Meij, B.S. Malnutrition Screening and Assessment in the Cancer Care Ambulatory Setting: Mortality Predictability and Validity of the Patient-Generated Subjective Global Assessment Short form (PG-SGA SF) and the GLIM Criteria. Nutrients 2020, 12, 2287. [CrossRef] [PubMed]

19. Shi, H.; Li, W.; Qi, Y. Nutrition Screening and Evaluation; People's Medical Publishing House: Beijing, China, 2014.

20. Stratton, R.J.; Hackston, A.; Longmore, D.; Dixon, R.; Price, S.; Stroud, M.; King, C.; Elia, M. Malnutrition in hospital outpatients and inpatients: Prevalence, concurrent validity and ease of use of the 'malnutrition universal screening tool'('MUST') for adults. Br. J. Nutr. 2004, 92, 799-808. [CrossRef]

21. Chen, L.K.; Woo, J.; Assantachai, P.; Auyeung, T.W.; Chou, M.Y.; Iijima, K.; Jang, H.C.; Kang, L.; Kim, M.; Kim, S.; et al. Asian Working Group for Sarcopenia: 2019 Consensus Update on Sarcopenia Diagnosis and Treatment. J. Am. Med. Dir. Assoc. 2020, 21, 300-307.e302. [CrossRef] [PubMed]

22. Cederholm, T.; Barazzoni, R.; Austin, P.; Ballmer, P.; Biolo, G.; Bischoff, S.C.; Compher, C.; Correia, I.; Higashiguchi, T.; Holst, M.; et al. ESPEN guidelines on definitions and terminology of clinical nutrition. Clin. Nutr. 2017, 36, 49-64. [CrossRef]

23. Maeda, K.; Ishida, Y.; Nonogaki, T.; Mori, N. Reference body mass index values and the prevalence of malnutrition according to the Global Leadership Initiative on Malnutrition criteria. Clin. Nutr. 2020, 39, 180-184. [CrossRef] [PubMed] 
24. Shrout, P.E. Measurement reliability and agreement in psychiatry. Stat. Methods Med. Res. 1998, 7, 301-317. [CrossRef] [PubMed]

25. Power, L.; Mullally, D.; Gibney, E.R.; Clarke, M.; Visser, M.; Volkert, D.; Bardon, L.; de van der Schueren, M.A.E.; Corish, C.A.; $\mathrm{MaNu}$, E.L.C. A review of the validity of malnutrition screening tools used in older adults in community and healthcare settings-A MaNuEL study. Clin. Nutr. ESPEN 2018, 24, 1-13. [CrossRef] [PubMed]

26. Yin, L.; Lin, X.; Zhao, Z.; Li, N.; He, X.; Zhang, M.; Yang, J.; Guo, Z.; Li, Z.; Wang, K.; et al. Is hand grip strength a necessary supportive index in the phenotypic criteria of the GLIM-based diagnosis of malnutrition in patients with cancer? Support. Care Cancer 2021, 29, 4001-4013. [CrossRef] [PubMed]

27. Zhang, Z.; Wan, Z.; Zhu, Y.; Zhang, L.; Zhang, L.; Wan, H. Prevalence of malnutrition comparing NRS2002, MUST, and PG-SGA with the GLIM criteria in adults with cancer: A multi-center study. Nutrition 2021, 83, 111072. [CrossRef] [PubMed]

28. Cereda, E.; Pedrazzoli, P.; Lobascio, F.; Masi, S.; Crotti, S.; Klersy, C.; Turri, A.; Stobaus, N.; Tank, M.; Franz, K.; et al. The prognostic impact of BIA-derived fat-free mass index in patients with cancer. Clin. Nutr. 2021, 40, 3901-3907. [CrossRef] 